\title{
Relaxed $\eta$ - $\alpha$ quasimonotone and application to the generalized variational-like inequality problem
}

Qiao Chen ${ }^{1 \dagger}$ and Jie Luo ${ }^{2 \dagger}$

"Correspondence:

115084448@qq.com

${ }^{1}$ College of Life Sciences,

Chongqing Normal University,

Chongqing, 401331, China

†Equal contributors

Full list of author information is

available at the end of the article

\section{Springer}

\begin{abstract}
In this paper, some new mappings called relaxed $\eta$ - $\alpha$ quasimonotone and a relaxed $\eta-\alpha$ properly quasimonotone operator are first introduced. The relationships between them are obtained. After this, the variational-like inequality problem and the relaxed Minty variational-like inequality problem are discussed by use of the proposed generalized monotone operators. Furthermore, we give the gap function of the two variational-like inequalities and two kinds of optimization problems. Finally, we point out that the two optimization problems are equivalent under some conditions.
\end{abstract}

MSC: $90 C 26 ; 90 C 30$

Keywords: relaxed $\eta$ - $\alpha$ quasimonotone; variational-like inequality; gap function; optimization problem

\section{Introduction}

As we know, variational inequality theory plays an important role in many fields, such as optimal control, mechanics, economics, transportation equilibrium, engineering sciences. It is well known that the role of generalized monotonicity of the operator in the variational inequality problem corresponds to the role of generalized convexity of the objective function in the mathematical programming problem. From this, the importance of the study of generalized monotonicity is evident.

In recent years, a number of authors have proposed many important generalizations of monotonicity. In [1], Karamardian and Schaible gave seven kinds of generalized monotone mappings which, in the case of gradient mappings, were related to a generalized convex function. In [2], Fang and Huang introduced a new concept of relaxed $\eta$ - $\alpha$ monotonicity and obtained the existence for variational-like inequalities with relaxed $\eta$ - $\alpha$ monotone mappings in a reflexive Banach space. Bai et al. [3] introduced relaxed $\eta$ - $\alpha$ pseudomonotone and established the existence for variational-like inequalities with relaxed $\eta-\alpha$ pseudomonotone mappings in a reflexive Banach space. In [4], a series of sufficient and necessary conditions were given that related the generalized invexity of the function $\theta$ with the generalized invex monotonicity of its gradient function $\nabla \theta$. In [5], Yang introduced a gap function for many generalized variational inequalities. The relationships between the generalized convexity of functions and generalized monotone operators also have been investigated by many authors (see [6-9]). Ansari et al. [10] considered different kinds of generalized vector variational-like inequality problems.

O2013 Chen and Luo; licensee Springer. This is an Open Access article distributed under the terms of the Creative Commons Attribution License (http://creativecommons.org/licenses/by/2.0), which permits unrestricted use, distribution, and reproduction in any medium, provided the original work is properly cited. 
Based on the results in [2,3] and [5], relaxed $\eta-\alpha$ quasimonotone, relaxed $\eta-\alpha$ properly quasimonotone are proposed in this paper. With a more weakly monotone assumption, the existence for variational-like inequalities with a relaxed $\eta-\alpha$ quasimonotone mapping in a reflexive Banach space is discussed. After this, by the gap function, the equivalence between two kinds of optimization problems is obtained.

The paper is organized as follows. In Section 2, some concepts, basic assumptions and preliminary results are presented. In Section 3, the existence for variational-like inequalities with relaxed $\eta-\alpha$ quasimonotone mappings in a reflexive Banach space is established. In Section 4, the gap function of the relaxed Minty variational-like inequality is given, and the optimization problem is studied with it.

\section{Definitions and preliminary}

In this paper, let $X$ be a reflexive Banach space, and dual space $X^{*}, K$ is the nonempty subset of $X$. Now we recall some basic definitions as follows.

Definition 2.1 [2] A mapping $T: K \rightarrow X^{*}$ is said to be relaxed $\eta-\alpha$ monotone if there exist a mapping $\eta: K \times K \rightarrow X$ and a function $\alpha: X \rightarrow R$ with $\alpha(t z)=t^{p} \alpha(z)$ for all $t>0$ and $z \in X$ such that

$$
\langle T x-T y, \eta(x, y)| \geq \alpha(x-y), \quad \forall x, y \in K,
$$

where $p>1$ is a constant.

Definition 2.2 [3] A mapping $T: K \rightarrow X^{*}$ is said to be relaxed $\eta$ - $\alpha$ pseudomonotone if there exist a mapping $\eta: K \times K \rightarrow X$ and a function $\alpha: X \rightarrow R$ with $\alpha(t z)=t^{p} \alpha(z)$ for all $t>0$ and $z \in X$ such that, for any $x, y \in K$, we have

$$
\langle T y, \eta(x, y)| \geq 0 \quad \Rightarrow \quad\langle T x, \eta(x, y)| \geq \alpha(x-y)
$$

where $p>1$ is a constant.

Based on this, we give the definition of relaxed $\eta-\alpha$ quasimonotone operator.

Definition 2.3 A mapping $T: K \rightarrow X^{*}$ is said to be relaxed $\eta$ - $\alpha$ quasimonotone if there exist a mapping $\eta: K \times K \rightarrow X$ and a function $\alpha: X \rightarrow R$ with $\alpha(t z)=t^{p} \alpha(z)$ for all $t>0$ and $z \in X$ such that, for any $x, y \in K$, we have

$$
\langle T y, \eta(x, y)\rangle>0 \Rightarrow\langle T x, \eta(x, y)\rangle \geq \alpha(x-y),
$$

where $p>1$ is a constant.

Special cases:

(1) If $\eta(x, y)=x-y, \forall x, y \in K$, and $\alpha(x-y) \equiv 0$, then (1) implies

$$
\langle T y, x-y\rangle>0 \quad \Rightarrow \quad\langle T x, x-y\rangle \geq 0, \quad \forall x, y \in K
$$

and $T$ is quasimonotone. 
(2) If $\alpha(x-y) \equiv 0$, then (1) implies

$$
\langle T y, \eta(x, y)\rangle>0 \Rightarrow\langle T x, \eta(x, y)\rangle \geq 0, \quad \forall x, y \in K
$$

and $T$ is invex quasimonotone.

Remark 2.1 From the above definition, we can see that the following relationships hold. Relaxed $\eta$ - $\alpha$ monotone $\Rightarrow$ relaxed $\eta-\alpha$ pseudomonotone $\Rightarrow$ relaxed $\eta$ - $\alpha$ quasimonotone.

Example 2.1 Let $K \in(-\infty,+\infty), T(x)=x^{2}, x \in R$,

$$
\begin{aligned}
& \eta(x, y)= \begin{cases}3(x-y), & x>y, \\
2(x-y), & x \leq y,\end{cases} \\
& \alpha(z)= \begin{cases}-z^{2}, & z>0, \\
z^{2}, & x \leq 0 .\end{cases}
\end{aligned}
$$

It is clear that the mapping $T$ is a relaxed $\eta-\alpha$ quasimonotone operator. But it is not a relaxed $\eta-\alpha$ pseudomonotone operator. If we let $y=0, x=-1,\langle T y, \eta(x, y)\rangle=0$, but $\langle T x, \eta(x, y)\rangle<\alpha(x-y)$, which is a contradiction.

Bai, in reference [3], proved that $T$ was relaxed $\eta-\alpha$ pseudomonotone, but not a relaxed $\eta-\alpha$ monotone mapping.

Definition 2.4 A mapping $T: K \rightarrow X^{*}$ is said to be relaxed $\eta$ - $\alpha$ properly quasimonotone if there exist a mapping $\eta: K \times K \rightarrow X$ and a function $\alpha: X \rightarrow R$ with $\alpha(t z)=t^{p} \alpha(z)$ for all $t>0$ and $z \in X$ such that, for any $x_{1}, \ldots, x_{n} \in K$ and $y \in \operatorname{cov}\left\{x_{1}, \ldots, x_{n}\right\}$, there exists $i \in\{1,2, \ldots, n\}$ such that

$$
\left\langle T x_{i}, \eta\left(x_{i}, y\right)\right\rangle \geq \alpha\left(x_{i}-y\right)
$$

Remark 2.2 When $\eta\left(x_{i}, y\right)=x_{i}-y, \alpha\left(x_{i}-y\right) \equiv 0, T$ is a properly quasimonotone mapping.

\section{Condition A [4]}

(1) The function $\eta: X \times X \rightarrow X$ is skew, i.e., $\eta(x, y)+\eta(y, x)=0, \forall x, y \in X$.

(2) The $\eta$ is an affine function in the first argument.

\section{Remark 2.3}

(i) When $\eta$ is a skew function, we have $\eta(x, x)=0$;

(ii) When $\eta$ is a skew function and an affine function in the first argument, $\eta$ is an affine function in the second argument;

(iii) There exists a function $\eta$, which satisfies Condition A, for example, $\eta(x, y)=3(x-y), \forall x, y \in X$.

Theorem 2.1 Suppose that the mapping $T: K \rightarrow X^{*}$ is relaxed $\eta-\alpha$ properly quasimonotone, and $\eta$ satisfies Condition $\mathrm{A}$, then $T$ is relaxed $\eta-\alpha$ quasimonotone. 
Proof Let $x, y \in K$, and

$$
\langle T y, \eta(x, y)\rangle>0 .
$$

Let $y_{t}=y+t(x-y), t \in(0,1) . T$ is relaxed $\eta-\alpha$ properly quasimonotone, we have

$$
\left\langle T x, \eta\left(x, y_{t}\right)\right\rangle \geq \alpha\left(x-y_{t}\right)
$$

or

$$
\left\langle T y, \eta\left(y, y_{t}\right)\right\rangle \geq \alpha\left(y-y_{t}\right) .
$$

From Condition A and (5), we obtain

$$
\langle T y, \eta(y, x)| \geq t^{p-1} \alpha(y-x), \quad p>1 .
$$

Let $t \rightarrow 0$, and we imply that

$$
\langle T y, \eta(y, x)\rangle \geq 0 \text {. }
$$

With Condition A and (3),

$$
\langle T y, \eta(y, x)\rangle<0 \text {. }
$$

It is a contradiction with (7), so (5) is not true, and (4) is correct. In (4), let $t \rightarrow 0$, and we have

$$
\langle T x, \eta(x, y)| \geq \alpha(y-x)
$$

So, $T$ is relaxed $\eta-\alpha$ quasimonotone. The proof is completed.

\section{Variational-like inequality problem (VLIP)}

In this section, we discuss the following variational inequality problem.

(i) Variational-like inequality problem (VLIP):

Find $x \in K$ such that $\langle T x, \eta(x, y)\rangle \geq 0, \forall y \in K$, we denote by $S(T, K)$ the set of solutions.

(ii) Minty variational-like inequality problem (MVLIP):

Find $x \in K$ such that $\langle T y, \eta(x, y)\rangle \geq 0, \forall y \in K$, we denote by $\operatorname{SM}(T, K)$ the set of solutions.

(iii) Local Minty variational-like inequality problem (LMXLIP):

Find $x \in K$ such that $\langle T y, \eta(x, y)\rangle \geq 0$, there exists a neighborhood $U$ of $x$, $\forall y \in K \cap U$, we denote by $\operatorname{LM}(T, K)$ the set of solutions.

Now we give a new generalized variational-like inequality.

(iv) Relaxed Minty variational-like inequality problem (RMXLIP):

Find $x \in K$ such that $\langle T y, \eta(x, y)\rangle \geq \alpha(y-x), \forall y \in K$, we denote by $R M(T, K)$ the set of solutions. 
Definition 3.1 A function $F: K \rightarrow 2^{X}$ is called a KKM-function if for every finite subset $\left\{x_{1}, x_{2}, \ldots, x_{n}\right\}$ of $X$, the convex hull $\operatorname{conv}\left(\left\{x_{1}, x_{2}, \ldots, x_{n}\right\}\right) \subset \bigcap_{i=1}^{n} F\left(x_{i}\right)$.

Lemma 3.1 [3] Let a nonempty subset $K \subset X$ and a KKM-function $F: K \rightarrow 2^{X}$, if $F(x)$ is a compact set, $\forall x \in K$, then $\bigcap_{x \in K} F(x) \neq \emptyset$.

Theorem 3.1 Let $K$ be a nonempty convex subset of a real reflexive Banach space $X$, if $T$ : $K \rightarrow X_{*}$ is hemicontinuous and $\eta$ is a weak ${ }^{*}$ compact-valued, which satisfies Condition A, then $R M(T, K) \subset S(T, K)$.

Proof Suppose that $x \in R M(T, K)$, but $x \notin S(T, K)$, so there exists a point $y \in K$ such that $\langle T x, \eta(x, y)\rangle<0$. Since $T x$ is weak* compact-valued, there exists $\varepsilon>0$ such that

$$
\langle T x, \eta(x, y)\rangle<-\varepsilon
$$

Let set $V=\left\{x^{*} \in X^{*}:\left\langle x^{*}, \eta(y, x)\right\rangle<-\varepsilon\right\}, v$ is a weak* compact set, and $T x \in V$.

Let $x_{t}=t y+(1-t) x, t \in(0,1], T$ is hemicontinuous, there exists $\delta>0$ such that $T\left(x_{t}\right) \in V$, $\forall t \in(0, \delta)$.

That is,

$$
\left\langle T x_{t}, \eta(x, y)\right\rangle<-\varepsilon, \quad \forall t \in(0, \delta)
$$

On the other hand, $x \in R M(T, K)$, so for all $t \in(0, \delta)$, we have

$$
\left\langle T x_{t}, \eta\left(x_{t}, x\right)\right\rangle=t\left|T x_{t}, \eta(x, y)\right| \geq \alpha\left(x_{t}-x\right)=t^{p} \alpha(y-x) .
$$

That is,

$$
\left\langle T x_{t}, \eta(x, y)\right\rangle \geq t^{p} \alpha(y-x)
$$

When $t \rightarrow 0$, (8) and (9) contradict, so $R M(T, K \subset S(T, K))$.

Theorem 3.2 Let $K$ be a nonempty convex subset of the real reflexive Banach space $X$. Let $T: K \rightarrow X^{*}$ be relaxed $\eta-\alpha$ quasimonotone, $\eta$ satisfy Condition $\mathrm{A}$, and suppose that $\langle T x, \eta(x, y)\rangle, \alpha(x-y)$ is the continuity of $y$. Then one of the following assertions holds:

(i) $T$ is relaxed $\eta$ - $\alpha$ properly quasimonotone;

(ii) $\operatorname{LM}(T, K) \neq \emptyset$.

Proof Suppose that $T$ is not relaxed $\eta-\alpha$ properly quasimonotone, then there exist $x_{1}, x_{2}, \ldots, x_{n} \in K$ and $y \in \operatorname{conv}\left\{x_{1}, x_{2}, \ldots, x_{n}\right\}$ such that

$$
\left\langle T x_{i}, \eta\left(x_{i}, y\right)\right\rangle<\alpha\left(x_{i}-y\right), \quad \forall i \in\{1, \ldots, n\}
$$

By the continuity of $y$, there exists a neighborhood $U$ of $y$. For any $z \in U \cap K$, one has

$$
\left\langle T x_{i}, \eta\left(x_{i}, z\right)\right\rangle<\alpha\left(x_{i}-z\right), \quad \forall i \in\{1, \ldots, n\} .
$$


By relaxed $\eta-\alpha$ quasimonotone,

$$
\left\langle T z, \eta\left(x_{i}, z\right)\right\rangle<0, \quad \forall i \in\{1, \ldots, n\}
$$

From $y \in \operatorname{conv}\left\{x_{1}, x_{2}, \ldots, x_{n}\right\}$ and Condition A, it follows easily that

$$
\langle T z, \eta(y, z)\rangle \leq 0, \quad z \in U \cap K
$$

By Condition A, we have

$$
\langle T z, \eta(z, y)\rangle \leq 0, \quad z \in U \cap K
$$

That is, $y \in L M(T, K), L M(T, K) \neq \emptyset$.

When (ii) is not true, we could get (i) similarly.

Theorem 3.3 Let $K$ be a nonempty, compact, and convex subset of a real reflexive Banach space $X$. Let $T: K \rightarrow X^{*}$ be relaxed $\eta$ - $\alpha$ quasimonotone and upper hemicontinuous with weakly* compact values, and let $\eta$ satisfy Condition A. Then $S(T, K) \neq \emptyset$.

Proof According to Theorem 3.2, we have either $L M(T, K) \neq \emptyset$ or $T$ is relaxed $\eta$ - $\alpha$ properly quasimonotone. If $L M(T, K) \neq \emptyset$, similarly to reference [6], we can get $S(T, K) \neq \emptyset$.

On the other hand, if $T$ is relaxed $\eta$ - $\alpha$ properly quasimonotone, define the set-valued mapping $G: K \rightarrow 2^{X^{*}}$ by

$$
G(x)=\{y \in K:\langle T x, \eta(x, y)\rangle \geq \alpha(x-y)\} .
$$

For every $x_{1}, \ldots, x_{n} \in K$, and $y \in \operatorname{conv}\left\{x_{1}, \ldots, x_{n}\right\}$, relaxed $\eta$ - $\alpha$ properly quasimonotone implies that $y \in \bigcup_{i=1}^{n} G\left(x_{i}\right)$. In addition, for each $x \in K, G(x)$ is closed, $K$ is compact, so for each $x \in K, G(x)$ is compact. By Lemma 3.1, we obtain $\bigcap_{x \in K} G(x) \neq \emptyset$, which implies that $R M(T, K) \neq \emptyset$. Finally, by Theorem 3.1 , we get the result $S(T, K) \neq \emptyset$.

\section{Gap function and application to the mathematical programming problem}

In this section, we discuss the gap function of the relaxed Minty variational-like inequality, and use it to study the optimization problem.

Definition 4.1 [5] A function $\phi: K \rightarrow R$ is said to be a gap function for the variational inequality (VI) if it satisfies the following properties:

(i) $\phi(x) \leq 0, \forall x \in K$;

(ii) $\phi\left(x_{0}\right)=0$ if and only if $x_{0}$ solves (VI).

For the variational-like inequality $\langle T x, \eta(y, x)\rangle \geq 0, \forall y \in K$, Yang in reference [5] gives its gap function:

$$
\phi_{1}(x)=\min _{y \in K}\langle T x, \eta(y, x)\rangle
$$


He pointed out that the solution of the variational-like inequality was the solution of the following optimization problem:

$$
\left(\mathrm{P}_{1}\right) \quad\left\{\begin{array}{l}
\max \phi_{1}(x), \\
\text { s.t. } \quad x \in K .
\end{array}\right.
$$

Now we give the gap function of the relaxed Minty variational-like inequality as follows:

$$
\phi_{2}(x)=\min _{y \in K}\langle T y, \eta(y, x)\rangle-\alpha(y-x)
$$

Theorem 4.1 Let $\eta(x, x)=0, \alpha(0)=0$. Then $\phi_{2}(x)$ is the gap function of the relaxed Minty variational-like inequality.

Proof (i) For $x \in K$, then

$$
\phi_{2}(x)=\min _{y \in K}\langle T y, \eta(y, x)\rangle-\alpha(y-x) \leq\langle T x, \eta(x, x)\rangle-\alpha(x-x)=0
$$

(ii) If $\phi_{2}(x)=0$, then

$$
\begin{aligned}
& \min _{y \in K}\left\langle T y, \eta\left(y, x_{0}\right)\right\rangle-\alpha\left(y-x_{0}\right)=0, \\
& \left\langle T y, \eta\left(y, x_{0}\right)\right\rangle \geq \alpha\left(y-x_{0}\right), \quad \forall y \in K .
\end{aligned}
$$

That is, $x_{0} \in R M(T, K)$.

Conversely, if $x_{0} \in R M(T, K)$, then $\left\langle T y, \eta\left(y, x_{0}\right)\right\rangle-\alpha\left(y-x_{0}\right) \geq 0, \forall y \in K$. So, $\phi_{2}\left(x_{0}\right) \geq 0$, by $\phi_{2}\left(x_{0}\right) \leq 0$, we can get $\phi_{2}\left(x_{0}\right)=0$. The proof is completed.

For the relaxed Minty variational-like inequality, we discuss the following optimization problem:

$$
\left(\mathrm{P}_{2}\right) \quad\left\{\begin{array}{l}
\max \phi_{2}(x), \\
\text { s.t. } \quad x \in K .
\end{array}\right.
$$

It is clear that the solution of the relaxed Minty variational-like inequality is also the solution of $\left(\mathrm{P}_{2}\right)$.

Finally, we discuss the relationship between the problems of $\left(\mathrm{P}_{1}\right)$ and $\left(\mathrm{P}_{2}\right)$.

Theorem 4.2 Let $K$ be a nonempty closed convex subset of the real reflexive Banach space $X$, and let $T: K \rightarrow X^{*}$ be hemicontinuous and relaxed $\eta-\alpha$ pseudomonotone. Assume that:

(i) $\eta(x, x)=0$ for all $x$ in $K$;

(ii) for any fixed $y, z$ in $K$, the mapping $x \longmapsto\langle T z, \eta(x, y)\rangle$ is convex.

Then the problems of $\left(\mathrm{P}_{1}\right)$ and $\left(\mathrm{P}_{2}\right)$ are equivalent.

Proof From Theorem 3.1 in reference [3], we know that the solution of the variational-like inequality and the solution of the relaxed Minty variational-like inequality are equivalent. So, we can get that the problems of $\left(\mathrm{P}_{1}\right)$ and $\left(\mathrm{P}_{2}\right)$ are equivalent. 


\section{Competing interests}

The authors declare that they have no competing interests.

\section{Authors' contributions}

All authors contributed equally and significantly in writing this article. All authors read and approved the final manuscript.

\section{Author details}

${ }^{1}$ College of Life Sciences, Chongqing Normal University, Chongqing, 401331, China. ${ }^{2}$ Mathematics Teaching and Research Group, Sichuan Dazhu Middle School, Sichuan, 635100, China.

\section{Acknowledgements}

The authors are thankful to Professor Xin Min Yang for his teaching and valuable comments on the original version of this paper. This research is partially supported by the National Natural Science Foundation of China (11201240).

Received: 22 January 2013 Accepted: 10 October 2013 Published: 07 Nov 2013

\section{References}

1. Karamardian, S, Schaible, S: Seven kinds of monotone maps. J. Optim. Theory Appl. 66, 37-46 (1990)

2. Fang, YP, Huang, NJ: Variational-like inequalities with generalized monotone mappings in Banach spaces. J. Optim. Theory Appl. 118, 327-338 (2003)

3. Bai, MR, Zhou, SZ, Ni, GY: Variantional-like inequalities with relaxed pseudomonotone mappings in Banach spaces. Appl. Math. Lett. 19, 547-554 (2006)

4. Ruiz-Garzon, G, Osuna-Gomez, R, Rufian-Lizana, A: Generalized invex monotonicity. Eur. J. Oper. Res. 144, 501-512 (2003)

5. Yang, XQ: On the gap functions of prevariational inequalities. J. Optim. Theory Appl. 116, 437-452 (2003)

6. Aussel, D, Hadjisavvas, N: On quasimonotone variational inequalities. J. Optim. Theory Appl. 121, $445-450$ (2004)

7. Yang, XM, Yang, XQ, Teo, KL: Generalized invexity and generalized invariant monotonicity. J. Optim. Theory Appl. 117, 607-625 (2003)

8. Jabarootian, T, Zafarani, J: Generalized invariant monotonicity and invexity of nondifferentiable functions. J. Glob. Optim. 36, 537-564 (2006)

9. Bai, MR, Hadjisavvas, N: Relaxed quasimonotone operators and relaxed quasiconvex functions. J. Optim. Theory Appl. 138, 329-339 (2008)

10. Ansari, QH, Rezaie, M, Zafarani, J: Generalized vector variational-like inequalities and vector optimization. J. Glob. Optim. 53, 271-284 (2012)

Cite this article as: Chen and Luo: Relaxed $\eta-\alpha$ quasimonotone and application to the generalized variational-like inequality problem. Journal of Inequalities and Applications 2013, 2013:488

\section{Submit your manuscript to a SpringerOpen ${ }^{\circ}$ journal and benefit from:}

- Convenient online submission

- Rigorous peer review

Immediate publication on acceptance

Open access: articles freely available online

- High visibility within the field

- Retaining the copyright to your article 